\title{
Does Aerobic Vaginitis Have Adverse Pregnancy Outcomes? Prospective Observational Study
}

\author{
Mahmoud F. Hassan $\mathbb{D}^{1},{ }^{1}$ Nancy M. A. Rund $\mathbb{D}^{1,2}$ Osama El-Tohamy, ${ }^{1}$ Mahmoud Moussa, \\ Yahia Z. Ali, ${ }^{3}$ Nehal Moussa, ${ }^{4}$ Ahmed A. Abdelrazik, ${ }^{5}$ and Enas A. A. Abdallah ${ }^{6}$ \\ ${ }^{1}$ Department of Obstetrics and Gynecology, Faculty of Medicine, Ain Shams University, Cairo, Egypt \\ ${ }^{2}$ Department of Obstetrics and Gynecology, Bugshan Hospital, Jeddah, Saudi Arabia \\ ${ }^{3}$ Department of Obstetrics and Gynecology, Faculty of Medicine, Fayoum University, Fayoum, Egypt \\ ${ }^{4}$ Laboratory Department, Ministry of Interior Hospitals, Cairo, Egypt \\ ${ }^{5}$ Department of Obstetrics and Gynecology, Nasser Institute for Research and Treatment, Cairo, Egypt \\ ${ }^{6}$ Department of Pediatrics and Neonatology, Faculty of Medicine, Cairo University, Cairo, Egypt
}

Correspondence should be addressed to Mahmoud F. Hassan; mahmoudfathy74@yahoo.com

Received 14 June 2019; Revised 2 November 2019; Accepted 19 December 2019; Published 18 January 2020

Academic Editor: Bryan Larsen

Copyright (C) 2020 Mahmoud F. Hassan et al. This is an open access article distributed under the Creative Commons Attribution License, which permits unrestricted use, distribution, and reproduction in any medium, provided the original work is properly cited.

\begin{abstract}
Background. Aerobic vaginitis (AV) is an aberration within the balanced vaginal microbiota. Only few reports have documented the adverse pregnancy outcomes related to AV. Nonetheless, the exact role of AV in pregnancy and the potential benefit of its screening need further study. Our goal was to evaluate the association between aerobic vaginitis (AV) in late pregnancy and maternal and neonatal outcomes. Methods. In this prospective observational study, a total of 600 singleton pregnant women with intact fetal membranes at a gestational age of 34-36 weeks were recruited (one hundred women with AV and 500 pregnant women without AV). The study protocol excluded patients with other forms of vaginal infection. Pregnancy outcomes were traced and documented. The primary outcome was the association between AV and preterm labor. The current study compared the maternal and neonatal outcomes among pregnant women with and without AV in unadjusted and adjusted analyses with the odds ratio (OR) and 95\% confidence interval (CI) reported. Results. There was an association between AV and with preterm birth (adjusted OR 3.06, 95\% CI 1.58-5.95) and prelabor rupture of membranes (adjusted OR 6.17, 95\% CI 3.24-11.7). For neonatal outcomes, AV was associated with a higher incidence of neonatal ICU admission (adjusted OR 2.19, 95\% CI 1.1-4.34). Severe forms of AV significantly increased the incidence of PTB $(p=0.0014)$ and PROM ( $p=0.0094)$ when compared to less severe forms of AV. Conclusion. AV is common in late pregnancy and is linked to a diversity of adversative pregnancy outcomes including preterm birth, PROM, and neonatal ICU admission. Moreover, the incidence of PTB and PROM might further increase with the severity of AV. Clinicians should pay more consideration to vaginal microbiota assessment during pregnancy.
\end{abstract}

\section{Introduction}

The vaginal bacterial flora consists of many microbial species, principally Lactobacillus species. The balance and interactions among vaginal microbes are critical for a healthy vaginal microenvironment [1]. Physiological changes during pregnancy result in vaginal mucosal congestion and hypertrophy, which benefit the growth of pathogenic microorganisms within the vagina [2]. The definition of aerobic vaginitis
(AV) is a clinical entity first proposed by Donders et al. in 2002 [3]. Aerobic vaginitis is an aberration within the balanced vaginal bacterial flora. Aerobic vaginitis is characterized by abnormal vaginal microflora accompanied by an increased localized inflammatory reaction and immune response [4]. The prevalence of AV varies from 5 to $10.5 \%$ among symptomatic nonpregnant women [5] and 4 to $8 \%$ during pregnancy [6]. Concerns about AV have been raised because it tends to involve mixed infections [7]. If 
undiagnosed or untreated, AV might interfere with female reproductive health and result in perinatal complications, such as preterm birth (PTB), prelabor rupture of membranes (PROM), and fetal infection $[8,9]$.

Notwithstanding the association between AV and pregnancy outcomes has been investigated in several studies, AV remains incompletely understood [10]. Previous epidemiological studies recognized a high incidence of an unexpected pregnancy outcome and premature birth due to $\mathrm{AV}$ in Africa and worldwide $[10,11]$. In attempts to explore the association between AV in late pregnancy and adverse perinatal outcomes, we conducted this prospective, observational, and powered study.

\section{Materials and Methods}

We conducted the present prospective observational study at the Obstetrics and Gynecology Department, Bugshan Hospital, Jeddah, Saudi Arabia, from February 2013 till November 2018. The Local Institutional Review Board and Ethics Committee granted the study protocol before study commencement. All participants provided written consent before inclusion. Our study followed the ethical standards of the Declaration of Helsinki. All pregnant women at a period of gestation 34-36 weeks as calculated by the last menstrual period or the first trimester ultrasound diagnosed with AV were consecutively enrolled. Participants were selected among pregnant women who attended for routine antenatal care in the outpatient clinic. We excluded women with history of previous preterm labor or threatened preterm labor, multiple gestation, women with rupture of membranes, antepartum hemorrhage, structural and functional abnormalities of the uterus, other specific vaginal infection including vulvovaginal candidiasis (VVC), bacterial vaginosis (BV), Trichomonas vaginalis (TV), or mixed vaginal infections ( $\geq 2$ types of simultaneous vaginal infection), and induced preterm labor for any obstetrical and medical condition.

At enrollment, all participants had detailed history, clinical examination, and a detailed transabdominal sonography. Then, participants underwent gynecological examinations and vaginal discharge collection. A nonlubricated sterile speculum was inserted before any other vaginal examination was made. Vaginal $\mathrm{pH}$ was evaluated by color strips. We obtained samples of vaginal discharge from the upper lateral vaginal wall using long cotton swabs. Samples were spread in the clinic onto three slides and were mixed with a drop of saline on one slide and a drop of $10 \%$ potassium hydroxide $(\mathrm{KOH})$ on a second slide; the third slide was Gram-stained. Then, all slides were sent for immediate microscopic examination. Also, one vaginal swab sample was sent to the laboratory for further aerobic culturing aimed at detection of aerobic bacterial growth.

Aerobic vaginitis was diagnosed when a composite AV score $\geq 3$ was determined by saline wet mount microscopy [3]. We used Nugent's criteria to diagnose BV based on Gram stain assessment [12]. Candidiasis was determined by direct observation of hyphae or budding yeast on a $10 \%$ potassium hydroxide preparation slide [13]. Trichomonas vaginalis was diagnosed when TV was microscopically distinguished in the saline wet mount smear [14]. All smears were examined by the same microscopist who was blinded for the patient's data. Confirmed cases with lone AV and control participants were followed up to evaluate pregnancy outcomes.

The primary outcome was preterm birth (defined as delivery before 37 weeks). Secondary outcome measures encompassed the following maternal outcomes: prelabor rupture of membranes (PROM) (defined as rupture of membranes before the onset of labor), preterm PROM (defined as rupture of membranes before 37 weeks), chorioamnionitis, cesarean delivery, and puerperal sepsis. The following neonatal outcomes were considered: low birth weight (defined as birth weight $<10$ th percentile for gestational age) [15], neonatal jaundice, neonatal sepsis (confirmed with a positive culturing for microorganisms from a sample of CSF, blood, or urine) [16], neonatal asphyxia (defined as (1) arterial cord $\mathrm{pH}<7.0$, (2) Apgar score of 3 or less for greater than 5 minutes, (3) evidence of altered neurological status, and (4) multisystem organ injury or failure) [17], neonatal intensive care unit (NICU) admission, stillbirth (defined as a baby born with no signs of life at or after 28 weeks of gestation) [18], and neonatal death (defined as deaths among live births during the first 28 completed days of life) [18].

The perinatal outcomes were compared between the offspring of patients with aerobic vaginitis and without AV. Baseline clinical characteristics between women with AV and without AV were compared using the chi-squared test or Fisher exact test for categorical variables and the MannWhitney $U$ test or Student $t$-test for continuous variables, as appropriate. Odds ratios (ORs) and $95 \%$ confidence intervals (CIs) were calculated for the outcomes of interests. Multivariate logistic regression was used to adjust for patients' age, BMI, parity, previous cesarean delivery, hypertensive disorders with pregnancy, pregestational and gestational diabetes mellitus, smoking habits, education level, and woman's occupation. Normality of distribution of the continuous variables was evaluated using the Shapiro-Wilk test. We tested final models with the Hosmer-Lemeshow goodness-of-fit test. All analyses were performed using the Statistical Package for the Social Sciences, version 14.0 (SPSS Inc., Chicago, IL, USA), and GraphPad Prism, version 6 (GraphPad Software Inc., La Jolla, CA, USA).

A priori sample size estimation was performed using PS: Power and Sample Size Calculation, version 3.1.6 (Department of Biostatistics, Vanderbilt University, Nashville, TN, USA). We planned a study of independent cases and controls with five controls per one case to study the association between $\mathrm{AV}$ and preterm birth (primary outcome). Prior data show that the PTB rate among controls was $9.2 \%$ and $19.7 \%$ for experimental subjects [19]. Thus, we need to study at least 95 experimental subjects and 475 control subjects to reject the null hypothesis that the PTB rates for experimental and control subjects are equal with a power of $80 \%$ and a type I error of 0.05 .

\section{Results}

Figure 1 represents the patient flow chart. Initial recruitment included 1,328 women. Twenty-three women declined to 


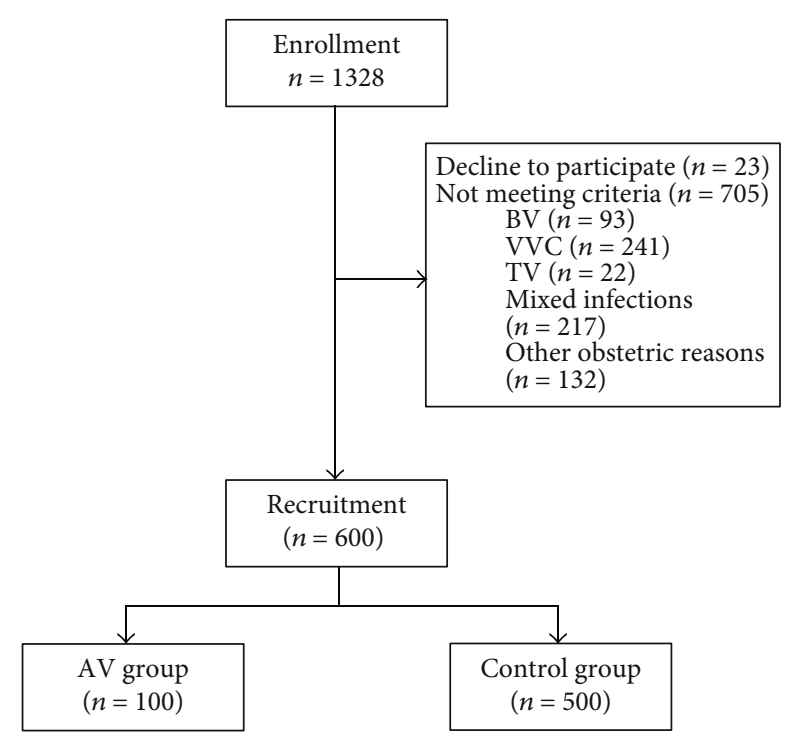

Figure 1: Patient flow chart. BV: bacterial vaginosis; VVC: vulvovaginal candidiasis; TV: Trichomonas vaginalis; AV: aerobic vaginitis.

participate in the study. Meanwhile, 705 women did not match the criteria for participation in our study.

Several clinical characteristics were similar between pregnant women with aerobic vaginitis and pregnant women without AV in our cohort including age, BMI, parity, previous cesarean delivery rate, hypertensive disorders with pregnancy, pregestational and gestational diabetes mellitus, and smoking habits. However, a few characteristics have differed between both groups. Women with AV were less likely to have college-level education or above and were more likely to be not working (Table 1).

In unadjusted analysis, there was an association between women with AV and preterm birth (18\% vs. $6.8 \%)$ and prelabor rupture of membranes (24\% vs. $5.4 \%)$ and neonatal asphyxia (6\% vs. $1.6 \%$ ) compared with women without AV (Table 2). In multivariate analysis, pregnant women with AV remained associated with preterm birth (adjusted OR $3.06,95 \%$ CI 1.58-5.95) and prelabor rupture of membranes (adjusted OR 6.17, 95\% CI 3.24-11.7) (Table 2).

For neonatal outcomes, pregnant women with AV were associated with neonatal asphyxia (5\% vs. $1.6 \%$ ) and neonatal ICU admission (15\% vs. $7.2 \%$ ) compared with pregnant women without AV in unadjusted analysis (Table 3). In the adjusted analysis, women with AV remained associated with neonatal ICU admission (adjusted OR 2.19, 95\% CI 1.1-4.34) compared with pregnant women without AV. However, women with AV were no longer significantly associated with neonatal asphyxia in the adjusted analysis (Table 3 ).

Aerobic culturing revealed that the most commonly identified pathogens among symptomatic women with a presumptive diagnosis of AV were Staphylococcus aureus, Enterococcus faecalis, Streptococcus agalactiae, and Escherichia coli. Other less frequent isolated pathogens were Staphylococcus saprophyticus, Lactobacillus acidophilus, Staphylococcus epidermidis, Corynebacterium, and Streptococcus pyogenes.
Table 4 provides information about the comparison of the maternal and neonatal outcomes according to the severity of AV. It indicates that the incidence of PTB and PROM is significantly increased with the severity of AV.

\section{Discussion}

The equilibrium and interactions between vaginal microbes are important to female vaginal health. Lactobacilli that dominate within the vaginas of most healthy women play a significant role in protecting the host from genital tract infections $[20,21]$. Aerobic vaginitis is a newly recognized vaginal flora disorder characterized by a shift from vaginal microbiota dominated by the Lactobacilli to an overgrowth of aerobic bacteria leading to adverse perinatal outcomes [22].

In our study, screening pregnant women for $\mathrm{AV}$ at 34-36 weeks of gestation declared higher odds for preterm labor, PROM, and neonatal ICU admission in pregnant women with AV than control participants. Moreover, severe forms of AV significantly increased the incidence of PTB and PROM. Ascending infection caused by vaginal microorganisms might justify the mechanism of AV-related adverse pregnancy outcomes. Some bacterial species in AV produce sialidases, which degrade host defense molecules such as IgA and can remove sialic acid from mucosal epithelial cells and mucins. The elimination of sialic acid from secretory IgA leads to IgA proteolysis and a lowered local immune response [23]. On top of that, AV probably linked to the increased concentrations of IL-1b, IL-6, and IL- 8 which are known risk factors for adverse pregnancy outcome $[6,24]$.

Many earlier studies have not recognized or ignored the contributing effect of AV on perinatal outcomes [25, 26]. However, new culture-based studies confirmed the association between AV and preterm birth $[4,27]$. On the contrary, a recent study by Han and coworkers did not show the association between AV and preterm labor. They incorporated cases with mixed vaginal infection along with many cases with solitary vaginal infection pathogens other than $\mathrm{AV}$ in their study; thus, they had a smaller population with only AV. Nevertheless, the same study was compatible with our findings concerning the association between AV and PROM. Han et al. found that pregnant women with AV were associated with a higher frequency for PROM $(p<0.05)$ [28]. Besides, a recent review article by Kaambo and Africa stated that even when asymptomatic, aerobic vaginitis may represent a risk factor for preterm delivery and PROM, ascending chorioamnionitis, and a neonatal mortality rate of $25 \%-90 \%$ because of congenital neonatal sepsis [29].

Our study has some limitations to consider. First, we screened pregnant women at late pregnancy (34-36 weeks). Earlier screening during pregnancy might detect more impacts of AV on perinatal outcomes. Second, the study was designed primarily to study only the association between AV and preterm labor and excluded other causes of vaginal infections. So, our study did not compare the impacts of AV to other forms of vaginal infections. Nonetheless, this study has the strength of being, to our knowledge, the first prospective powered study to evaluate the perinatal outcome of $\mathrm{AV}$. 
TABLe 1: Demographic and clinical characteristics.

\begin{tabular}{|c|c|c|c|}
\hline Variable & $\operatorname{AV}(n=100)$ & Control $(n=500)$ & $p$ value \\
\hline Age (years) & $27.9 \pm 0.57$ & $27.2 \pm 0.23$ & $0.227 *$ \\
\hline BMI $\left(\mathrm{kg} / \mathrm{m}^{2}\right)$ & $28.1 \pm 0.46$ & $28 \pm 0.2$ & $0.964 *$ \\
\hline Parity & $1(0-2)$ & $1(0-2)$ & $0.125 * *$ \\
\hline Previous cesarean delivery & $32(32 \%)$ & $154(30.8 \%)$ & $0.814^{\dagger}$ \\
\hline Hypertensive disorders with pregnancy & $4(4 \%)$ & $24(4.8 \%)$ & $1.0^{\ddagger}$ \\
\hline Pregestational and gestational diabetes mellitus & $7(7 \%)$ & $29(5.8)$ & $0.645^{\dagger}$ \\
\hline Smoking habits & $7(7 \%)$ & $33(6.6 \%)$ & $0.828^{\dagger}$ \\
\hline Education level & & & $<0.001^{\dagger}$ \\
\hline High school or less & $37(37 \%)$ & $66(13.2 \%)$ & \\
\hline College or above & $63(63 \%)$ & $434(86.8 \%)$ & \\
\hline Woman's occupation & & & $0.022^{\dagger}$ \\
\hline Not working & $34(34 \%)$ & $114(22.8 \%)$ & \\
\hline Working & $66(66 \%)$ & $386(77.2 \%)$ & \\
\hline
\end{tabular}

AV: aerobic vaginitis; BMI: body mass index. Data are presented as mean \pm standard deviation, median (interquartile range), or number (\%). $*$ Student's $t$-test was used; $* *$ Mann-Whitney $U$ test was used; ${ }^{\dagger}$ Chi-squared test was used; ${ }^{\ddagger}$ Fisher exact test was used. $p$ value $<0.05$ is significant.

TABle 2: Associations of maternal outcomes among pregnant women with aerobic vaginitis and without aerobic vaginitis.

\begin{tabular}{|c|c|c|c|c|c|c|}
\hline Variable & $\operatorname{AV}(n=100)$ & Control $(n=500)$ & $\mathrm{OR}(\mathrm{CI})$ & $p$ value & Adjusted $*$ OR $(\mathrm{CI})$ & $p$ value \\
\hline PTB & $18(18 \%)$ & $34(6.8 \%)$ & $3.0(1.62-5.58)$ & $<0.001$ & $3.06(1.58-5.95)$ & 0.001 \\
\hline PROM & $24(24 \%)$ & $27(5.4 \%)$ & $5.53(3.03-10.09)$ & $<0.001$ & $6.17(3.24-11.7)$ & $<0.001$ \\
\hline pPROM & $7(7 \%)$ & $21(4.2 \%)$ & $1.72(0.71-4.16)$ & 0.231 & $1.73(0.68-4.4)$ & 0.249 \\
\hline Chorioamnionitis & $2(2 \%)$ & $3(0.6 \%)$ & $3.38(0.56-20.5)$ & 0.185 & $5.87(0.9-38.24)$ & 0.064 \\
\hline Cesarean delivery & $32(32 \%)$ & $149(29.8 \%)$ & $1.11(0.7-1.76)$ & 0.662 & $1.11(0.68-1.8)$ & 0.679 \\
\hline Puerperal sepsis ${ }^{\dagger}$ & $1(1 \%)$ & 0 & & & & \\
\hline
\end{tabular}

AV: aerobic vaginitis; OR: odds ratio; CI: confidence interval; PTB: preterm birth; PROM: prelabor rupture of membranes; pPROM: preterm prelabor mature rupture of membranes. Data are presented as number $(\%)$ and odds ratio with confidence interval. $*$ The analysis was adjusted for maternal age, body mass index, parity, previous cesarean delivery, hypertensive disorders with pregnancy, pregestational and gestational diabetes mellitus, smoking habits, education level, and woman's occupation. ${ }^{\dagger}$ As a result of the low frequency of this outcome, odds ratio was not reported. $p$ value $<0.05$ is significant.

TABLE 3: Associations of neonatal outcomes among pregnant women with aerobic vaginitis and without aerobic vaginitis.

\begin{tabular}{|c|c|c|c|c|c|c|}
\hline Variable & $\operatorname{AV}(n=100)$ & Control $(n=500)$ & OR (CI) & $p$ value & Adjusted $*$ OR $(\mathrm{CI})$ & $p$ value \\
\hline Low birth weight & $8(8 \%)$ & $19(3.8 \%)$ & $2.2(094-5.18)$ & 0.071 & $2.13(0.85-5.4)$ & 0.109 \\
\hline Neonatal jaundice & $12(12 \%)$ & $38(7.6 \%)$ & $1.66(0.83-3.3)$ & 0.15 & $1.47(0.7-3.09)$ & 0.314 \\
\hline Neonatal sepsis & $2(2 \%)$ & $2(0.4 \%)$ & $5.08(0.71-36.51)$ & 0.106 & $4.99(0.6-41-53)$ & 0.137 \\
\hline Neonatal asphyxia & $5(5 \%)$ & $8(1.6 \%)$ & $3.24(1.04-10.11)$ & 0.043 & $2.9(0.85-9.9)$ & 0.089 \\
\hline NICU admission & $15(15 \%)$ & $36(7.2 \%)$ & $2.28(1.19-4.34)$ & 0.013 & $2.19(1.1-4.34)$ & 0.025 \\
\hline Stillbirth & 0 & 0 & & & & \\
\hline Neonatal death & $2(2 \%)$ & $1(0.2 \%)$ & $10.18(0.91-113.4)$ & 0.059 & $5.14(0.3-86.69)$ & 0.256 \\
\hline
\end{tabular}

AV: aerobic vaginitis; OR: odds ratio; CI: confidence interval; NICU: neonatal intensive care unit. Data are presented as number (\%) and odds ratio with confidence interval. *The analysis was adjusted for maternal age, body mass index, parity, previous cesarean delivery, hypertensive disorders with pregnancy, pregestational and gestational diabetes mellitus, smoking habits, education level, and woman's occupation. $p$ value $<0.05$ is significant.

\section{Conclusions}

In conclusion, aerobic vaginitis is a common form of vaginal infection during pregnancy. AV is associated with a high incidence of preterm labor, PROM, and neonatal ICU admission. Moreover, the incidence of PTB and PROM might further increase with the severity of AV. Vaginal microbiota screening at 34-36 weeks might contribute to the improvement of pregnancy outcomes and reduction in preterm labor, PROM, and other adverse pregnancy outcomes. Additionally, clinicians should pay more consideration to vaginal microbiota assessment during pregnancy. Larger observational studies 
TABLE 4: Comparison of maternal and neonatal outcomes according to the severity of aerobic vaginitis.

\begin{tabular}{lccc}
\hline Variable & $\begin{array}{c}\text { Severe AV } \\
(n=24)\end{array}$ & $\begin{array}{c}\text { Mild/moderate } \\
\text { AV }(n=76)\end{array}$ & $\begin{array}{c}p \\
\text { value }\end{array}$ \\
\hline PTB & 10 & $8(10.5 \%)$ & 0.0014 \\
PROM & $(41.7 \%)$ & & \\
pPROM & 11 & $13(17.1 \%)$ & 0.0094 \\
Chorioamnionitis & $1(4.2 \%)$ & $1(1.3 \%)$ & 0.4242 \\
Cesarean delivery & $9(37.5 \%)$ & $23(30.3 \%)$ & 0.4403 \\
Puerperal sepsis & $1(4.2 \%)$ & 0 & 0.240 \\
Low birth weight & $3(12.5 \%)$ & $5(6.6 \%)$ & 0.3688 \\
Neonatal jaundice & $3(12.5 \%)$ & $9(11.8 \%)$ & 0.2128 \\
Neonatal sepsis & $1(4.2 \%)$ & $1(1.3 \%)$ & 0.4242 \\
Neonatal asphyxia & $2(8.3 \%)$ & $3(3.9 \%)$ & 0.5910 \\
NICU admission & $6(25 \%)$ & $9(11.8 \%)$ & 0.2128 \\
Neonatal death & $1(4.2 \%)$ & $1(1.3 \%)$ & 0.4242 \\
\hline
\end{tabular}

AV: aerobic vaginitis; PTB: preterm birth; PROM: prelabor rupture of membranes; pPROM: preterm prelabor rupture of membranes; NICU: neonatal intensive care unit. Data are presented as number (\%). $p$ value $<$ 0.05 is significant. Severe aerobic vaginitis was diagnosed when a composite AV score $>6$ was determined by saline wet mount microscopy [3].

recruiting more patients of different ethnic populations are needed to support our findings and properly study all aspects of perinatal outcome.

\section{Data Availability}

The data used to support the findings of this study are available from the corresponding author upon request.

\section{Conflicts of Interest}

The authors declare that they have no conflict of interest.

\section{Authors' Contributions}

Mahmoud F. Hassan is responsible for project development, study design, and software. All authors participated in the data collection, data analysis, manuscript drafting, and manuscript revision. All authors approved the final manuscript.

\section{References}

[1] B. Larsen and G. R. Monif, "Understanding the bacterial flora of the female genital tract," Clinical Infectious Diseases, vol. 32, no. 4, pp. e69-e77, 2001

[2] J. A. Svare, H. Schmidt, B. B. Hansen, and G. Lose, "Bacterial vaginosis in a cohort of Danish pregnant women: prevalence and relationship with preterm delivery, low birthweight and perinatal infections," British Journal of Obstetrics and Gynaecology, vol. 113, no. 12, pp. 1419-1425, 2006.

[3] G. G. Donders, A. Vereecken, E. Bosmans, A. Dekeersmaecker, G. Salembier, and B. Spitz, "Definition of a type of abnormal vaginal flora that is distinct from bacterial vaginosis: aero- bic vaginitis," British Journal of Obstetrics and Gynaecology, vol. 109, no. 1, pp. 34-43, 2002.

[4] G. Donders, G. Bellen, and D. Rezeberga, "Aerobic vaginitis in pregnancy," British Journal of Obstetrics and Gynaecology, vol. 118, no. 10, pp. 1163-1170, 2011.

[5] G. S. Tansarli, E. K. Kostaras, S. Athanasiou, and M. E. Falagas, "Prevalence and treatment of aerobic vaginitis among nonpregnant women: evaluation of the evidence for an underestimated clinical entity," European Journal of Clinical Microbiology \& Infectious Diseases, vol. 32, no. 8, pp. 977-984, 2013.

[6] G. Donders, G. Bellen, S. Grinceviciene, K. Ruban, and P. Vieira-Baptista, "Aerobic vaginitis: no longer a stranger," Research in Microbiology, vol. 168, no. 9-10, pp. 845-858, 2017.

[7] A. Fan, Y. Yue, N. Geng, H. Zhang, Y. Wang, and F. Xue, “Aerobic vaginitis and mixed infections: comparison of clinical and laboratory findings," Archives of Gynecology and Obstetrics, vol. 287, no. 2, pp. 329-335, 2013.

[8] C. Han, W. Wu, A. Fan et al., "Diagnostic and therapeutic advancements for aerobic vaginitis," Archives of Gynecology and Obstetrics, vol. 291, no. 2, pp. 251-257, 2015.

[9] C. Marconi, G. G. Donders, L. F. Martin et al., "Chlamydial infection in a high risk population: association with vaginal flora patterns," Archives of Gynecology and Obstetrics, vol. 285, no. 4, pp. 1013-1018, 2012.

[10] R. Rampersaud, T. M. Randis, and A. J. Ratner, "Microbiota of the upper and lower genital tract," Seminars in Fetal and Neonatal Medicine, vol. 17, no. 1, pp. 51-57, 2012.

[11] C. Nardis, L. Mosca, and P. Mastromarino, "Vaginal microbiota and viral sexually transmitted diseases," Annals of Hygiene, vol. 25, no. 5, pp. 443-456, 2013.

[12] R. P. Nugent, M. A. Krohn, and S. L. Hillier, "Reliability of diagnosing bacterial vaginosis is improved by a standardized method of gram stain interpretation," Journal of Clinical Microbiology, vol. 29, no. 2, pp. 297-301, 1991.

[13] M. Ilkit and A. B. Guzel, "The epidemiology, pathogenesis, and diagnosis of vulvovaginal candidosis: a mycological perspective," Critical Reviews in Microbiology, vol. 37, no. 3, pp. 250-261, 2011.

[14] C. Ohlemeyer, L. Hornberger, D. Lynch, and E. Swierkosz, "Diagnosis of Trichomonas vaginalis in adolescent females: InPouch TV culture versus wet-mount microscopy," Journal of Adolescent Health, vol. 22, no. 3, pp. 205-208, 1998.

[15] C. C. Lim and T. Mahmood, "Obesity in pregnancy," Best Practice \& Research: Clinical Obstetrics \& Gynaecology, vol. 29, no. 3, pp. 309-319, 2015.

[16] J. R. Delanghe and M. M. Speeckaert, "Translational research and biomarkers in neonatal sepsis," Clinica Chimica Acta, vol. 451, pp. 46-64, 2015.

[17] American College of Obstetrics and Gynecology, "Executive summary: neonatal encephalopathy and neurologic outcome, second edition. Report of the American College of Obstetricians and Gynecologists' Task Force on Neonatal Encephalopathy," Obstetrics \& Gynecology, vol. 123, no. 4, pp. 896-901, 2014.

[18] World Health Organization, Neonatal and perinatal mortality country, regional and global estimates, Geneva, 2006February 2019, https://apps.who.int/iris/bitstream/handle/10665/ 43444/9241563206_eng.pdf?sequence=1.

[19] G. Donders, K. Van Calsteren, G. Bellen et al., "Predictive value for preterm birth of abnormal vaginal flora, bacterial 
vaginosis and aerobic vaginitis during the first trimester of pregnancy," BJOG: An International Journal of Obstetrics \& Gynaecology, vol. 116, no. 10, pp. 1315-1324, 2009.

[20] G. Reid, "Cervicovaginal microbiomes-threats and possibilities," Trends in Endocrinology \& Metabolism, vol. 27, no. 7, pp. 446-454, 2016.

[21] S. Kovachev, "Defence factors of vaginal lactobacilli," Critical Reviews in Microbiology, vol. 44, no. 1, pp. 31-39, 2018.

[22] N. Geng, W. Wu, A. Fan et al., "Analysis of the risk factors for aerobic vaginitis: a case-control study," Gynecologic and Obstetric Investigation, vol. 81, no. 2, pp. 148-154, 2016.

[23] W. G. Lewis, L. S. Robinson, J. Perry et al., "Hydrolysis of secreted sialoglycoprotein immunoglobulin A (IgA) in ex vivo and biochemical models of bacterial vaginosis," Journal of Biological Chemistry, vol. 287, no. 3, pp. 2079-2089, 2012.

[24] J. C. Carey and M. A. Klebanoff, "Is a change in the vaginal flora associated with an increased risk of preterm birth?," American Journal of Obstetrics \& Gynecology, vol. 192, no. 4, pp. 1341-1346, 2005.

[25] P. Vieira-Baptista, J. Lima-Silva, C. Pinto et al., "Bacterial vaginosis, aerobic vaginitis, vaginal inflammation and major Pap smear abnormalities," European Journal of Clinical Microbiology \& Infectious Diseases, vol. 35, no. 4, pp. 657-664, 2016.

[26] G. G. Donders, "Bacterial vaginosis during pregnancy: screen and treat?," European Journal of Obstetrics \& Gynecology and Reproductive Biology, vol. 83, no. 1, pp. 1-4, 1999.

[27] U. S. Sangkomkamhang, P. Lumbiganon, W. Prasertcharoensuk, and M. Laopaiboon, "Antenatal lower genital tract infection screening and treatment programs for preventing preterm delivery," Cochrane Database of Systematic Reviews, vol. 1, no. 2, article CD006178, 2015.

[28] C. Han, H. Li, L. Han et al., "Aerobic vaginitis in late pregnancy and outcomes of pregnancy," European Journal of Clinical Microbiology \& Infectious Diseases, vol. 38, no. 2, pp. 233239, 2019.

[29] E. Kaambo and C. W. J. Africa, "The threat of aerobic vaginitis to pregnancy and neonatal morbidity," African Journal of Reproductive Health, vol. 21, no. 2, pp. 108-118, 2017. 


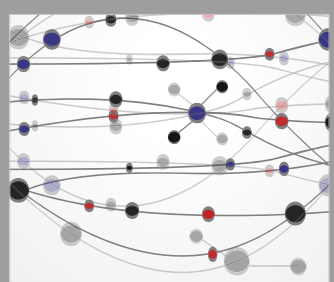

The Scientific World Journal
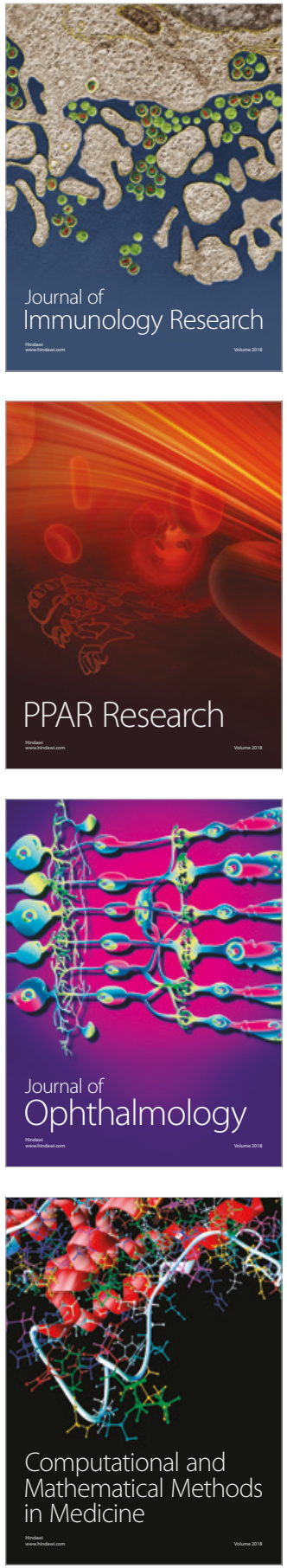

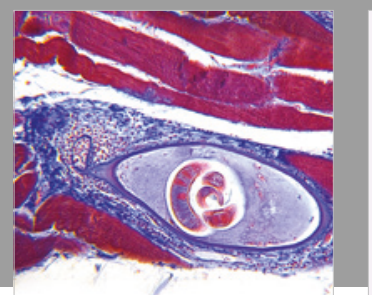

Gastroenterology Research and Practice

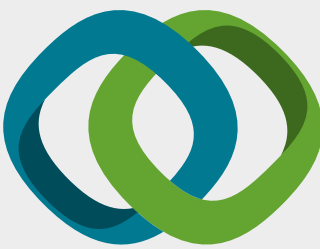

\section{Hindawi}

Submit your manuscripts at

www.hindawi.com
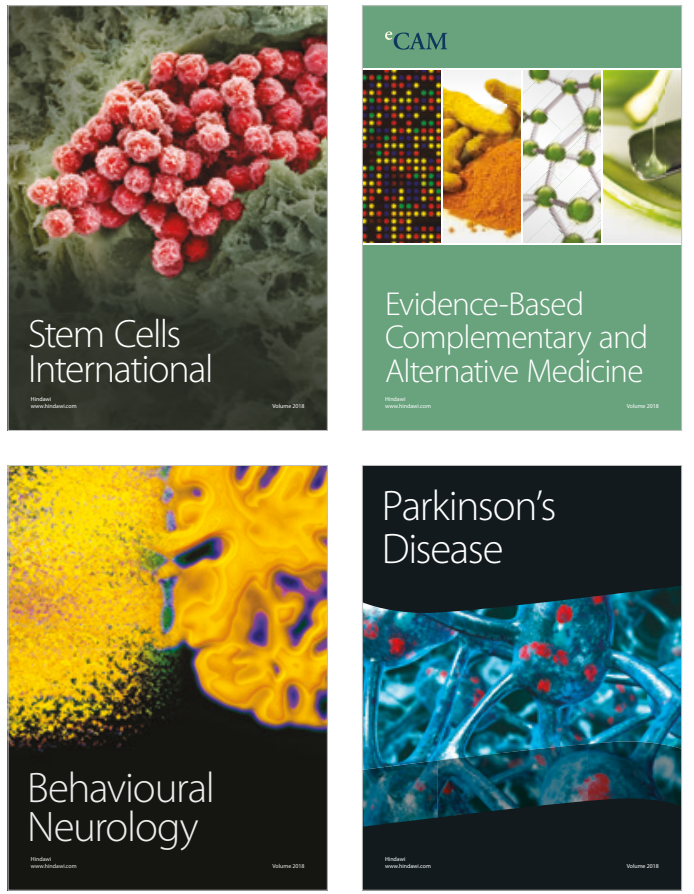

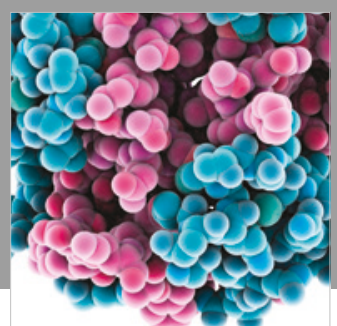

ournal of

Diabetes Research

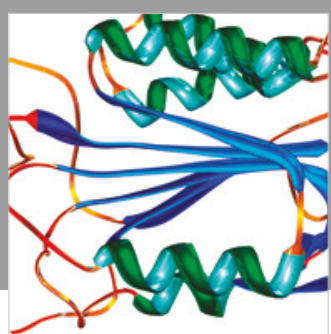

Disease Markers
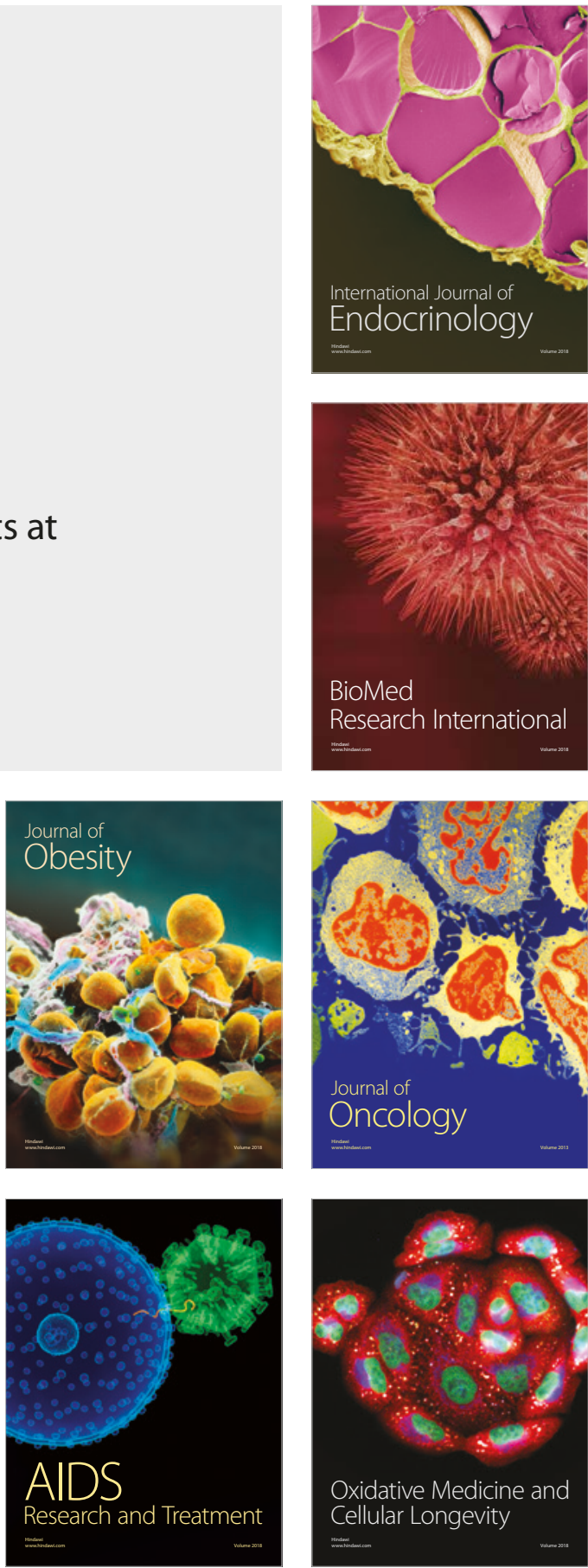